\title{
Breastfeeding: a reproductive factor able to reduce the risk of luminal $B$ breast cancer in premenopausal White women Fabiola Giudici $^{a, \star}$, Bruna Scaggiante $^{\mathrm{b}, \star}$, Serena Scomersi ${ }^{\mathrm{c}}$, Marina Bortul ${ }^{\mathrm{a}, \mathrm{c}}$, Maura Tonutti ${ }^{\mathrm{c}}$ and Fabrizio Zanconati ${ }^{\mathrm{a}, \mathrm{c}}$
}

In the medical literature, the role of breastfeeding and reproductive factors in the risk of breast carcinoma is still an open debate in premenopausal women. We highlight the role of breastfeeding and reproductive factors in luminal $A$ and luminal $B$, the most frequent breast cancers. This case-control study analyzes a White premenopausal population of 286 breast cancer patients, divided into molecular subtypes, and $\mathbf{5 7 8}$ controls matched by age. Multivariate logistic regression models were used to assess the relationships of breastfeeding and other reproductive factors (age at menarche, parity, age at first pregnancy, number of children) with the risk of breast cancers. Among the variables examined, reproductive factors did not alter the risk of cancer, whereas breastfeeding up to 12 months was a significant protective factor against luminal $B$ breast cancer (multivariate odds ratio: $0.22,95 \%$ confidence interval: $0.09-0.59, P=0.002$ ). In contrast, luminal A cases did not significantly correlate with breastfeeding or other

\section{Introduction}

Breast cancer is the leading cause of cancer death among women worldwide (Siegel et al., 2015). Several studies and meta-analyses have shown that reproductive factors are associated with the risk of breast cancer (Kelsey et al., 1993; Yang and Jacobsen, 2008; Butt et al., 2014). The underlying biological mechanisms are not yet clearly understood, but epidemiological evidence indicates that early menarche, older age at first full-term pregnancy, low parity, and lack of breastfeeding increase the risk of breast cancer (Kelsey et al., 1993; Collaborative Group on Hormonal Factors in Breast Cancer, 2002).

A protective effect of breastfeeding has been indicated (Collaborative Group on Hormonal Factors in Breast Cancer, 2002; Ursin et al., 2005; Ma et al., 2006; Horn et al., 2014), but the majority of published findings often present results for a mix of both premenopausal and postmenopausal women (Ursin et al., 2005; Butt et al., 2014) or only for postmenopausal women (Phipps et al., 2008; Horn et al., 2014). Recently, several studies have assessed the role of breastfeeding and other reproductive factors in different breast cancer subtypes, suggesting

This is an open-access article distributed under the terms of the Creative Commons Attribution-Non Commercial-No Derivatives License 4.0 (CCBY-NCND), where it is permissible to download and share the work provided it is properly cited. The work cannot be changed in any way or used commercially without permission from the journal. reproductive factors. Breastfeeding up to 12 months is strongly protective against the more aggressive luminal $B$, but not against the less aggressive luminal $A$ breast cancer in premenopausal White women. European Journal of Cancer Prevention 26:217-224 Copyright (C) 2017 The Author(s). Published by Wolters Kluwer Health, Inc.

European Journal of Cancer Prevention 2017, 26:217-224

Keywords: breast cancer risk, breastfeeding, luminal cancers, premenopausal White women, reproductive factors, retrospective study

Departments of a Medical, Surgical and Health Sciences, bife Sciences, University of Trieste and ${ }^{\mathrm{C}}$ Academic Hospital, Ospedali Riuniti, Trieste, Italy

Correspondence to Bruna Scaggiante, PhD, Department of Life Sciences, University of Trieste, Via Giorgeri, 1, 34127 Trieste, Italy

Tel: + 39040558 3686; fax: + 39040558 3691; e-mail: bscaggiante@units.it

*Fabiola Giudici and Bruna Scaggiante contributed equally to the writing of this article.

Received 30 September 2015 Accepted 9 January 2016

that the association of reproductive factors with the risk of breast cancer may vary according to breast cancer molecular profiles (Ursin et al., 2005; Phipps et al., 2008; Horn et al., 2014). The identification of which factors are related to the risk of breast cancer in populations of women identified by menopausal status, age, race, and molecular cancer subtypes still deserves further investigation (Warner et al., 2013; Horn et al., 2014). In particular, it remains to be clarified whether breastfeeding could exert a protective effect on breast cancers in premenopausal women (Negri et al., 1996; Ferlay et al., 2013; Butt et al., 2014).

This study investigates in a case-control population of White premenopausal women living in a country with a high breast cancer incidence the role of breastfeeding and reproductive factors on the risk of breast cancer stratified into molecular cancer subtypes. In particular, we analyzed the luminal breast cancers, accounting for about $75 \%$ of all breast cancers (Azim and Partridge, 2014; Rotunno et al., 2014).

\section{Patients and methods Study cohort}

We selected a homogeneous population of White premenopausal women living for at least 10 years in Trieste (Friuli-Venezia Giulia), an Italian region with the highest incidence of breast cancer: estimated age-standardized 
rates (ASRs) for Europe 94.2, ASRs for Italy 118.0, and ASRs for Friuli-Venezia Giulia 129.1 (European standard) (Ferlay et al., 2013; Zucchetto et al., 2013).

Premenopausal women were enrolled by Azienda Ospedaliera 'Ospedali Riuniti' of Trieste (Italy) between January 2006 and January 2014. The case patients had received a histopathologically confirmed first diagnosis of primary breast cancer between 2006 and 2013. The control participants were women who spontaneously joined breast cancer-prevention campaigns between 2009 and 2014; they had no breast diseases, which was confirmed by medical records. The cohort of women between 21 and 49 years of age were premenopausal (regular menstrual flow in the last year at the interview).

Cases were age adjusted and matched with two controls with an age interval of 5 years. The Mann-Whitney test confirmed the pairing.

\section{Data collection questionnaire}

Women were invited to fill out a questionnaire on demographics, reproductive history, breast pathology, BMI, and lifestyle. Reproductive history included questions on age an menarche, parity (defined by the number of full-term pregnancies), age at first full-term pregnancy, overall duration of breastfeeding ( $\leq 12$ months, $>12$ months), and menopausal status.

Questions on familial breast cancer history were included according to NICE guidelines (National Institute for Health and Care Excellence Pathway, created: October 2011, last updated: December 2013). Consent to the processing of data in an anonymous form for clinical research, epidemiology, and training (and with the aim of improving knowledge, treatment and prevention), in agreement with the Italian law art.81 D.lgs 196/2003, sez. $\mathrm{D}$, was obtained at the diagnosis of cases by the hospital. For each case and control, we obtained a signed informant consent during the interview to use the data in an anonymous form, in agreement with the Italian law art.81 D.lgs 196/2003, sez. D.

Physicians always conducted the structured questionnaire and the interviews lasted about $20 \mathrm{~min}$.

\section{Data collection on breast cancer and molecular profiles}

Histopathological features and data on estrogen receptor (ER), progesterone receptor (PR), and HER2 expression status were obtained from records. All invasive tumors were re-evaluated by pathologists to confirm aggressiveness and tumor type according to the WHO classification. Classification of molecular subtypes takes into account the expression of ER, PR, HER2, and Ki-67 according to St. Gallen 2013 (Goldhirsch et al., 2013).

All tests of ER, PR, and HER2 expression were based on immunohistochemistry testing. In equivocal HER2 cases, data from fluorescent in-situ hybridization assay records were used for molecular stratification. Patients with incomplete data on tumor markers were excluded from the study population. Breast cancer subtypes were grouped into the following categories: in-situ carcinomas, luminal A cases $(\mathrm{ER}+/ \mathrm{PR}+/ \mathrm{HER} 2-, \mathrm{Ki}-67<20 \%)$, luminal B cases (luminal B HER2 - : HER +/HER2 and at least one of $\mathrm{Ki}-67 \geq 20 \%$ or $\mathrm{PR}-$, luminal B HER2 + : ER +/HER2 + /any Ki-67/any PR), HER2 + (ER $-/ \mathrm{PR}-/ \mathrm{HER} 2+)$, and triple-negative breast cancers (ER - /PR - /HER2 - ).

The Ki-67 protein was evaluated on formalin-fixed tissue samples by the Joint Commission Accredited Hospital Laboratory using an automatic stainer (Lab Vision; Thermo Scientific, Runcorn, UK). The reaction with monoclonal antibody clone MIB-1 (Bio-Optica MILANO S.p.A, Milan, Italy; 1:200 dilution) was followed by HRP-DAB staining [UltraVision LP (Thermo Scientific); and DAB Quanto (Thermo Scientific)].

The HER2 monoclonal antibody was clone CB-11 (1:300 dilution; Aczon Biotech S.p.A, Bologna, Italy); the reactivity was evaluated using the detection kit HRP-DAB (Thermo Scientific). Every slide was evaluated by a pathologist on an optical microscope with a low magnification $(\times 10)$ and classified as positive, negative, or equivocal, in agreement with the guidelines of the American Society of Clinical Oncology and College of American Pathologists (ASCO-CAP, 2006; Wolff et al., 2007).

\section{Statistical analysis}

Continuous variables (age, age at menarche, and age at first full pregnancy) were analyzed using the Mann-Whitney test. In the univariate analysis, associations between reproductive factors and breast cancer were evaluated using the $\chi^{2}$-test. Multiple logistic regression was used to calculate odds ratios (ORs) and 95\% confidence intervals (CIs) as measures of the relative risk of cancers adjusted for age, age at menarche, BMI, oral contraceptive use, benign breast disease, and family history of breast cancer.

We fited different multivariate models: all cancer cases (independent of molecular subtype) versus controls, and luminal A and luminal B cases versus controls (selected at random within 5 -year age strata among 578 controls of the study).

Statistical analyses were carried out using R (the R Foundation for Statistical Computing; Version 3.0.3) and $P$-values less than 0.05 were considered statistically significant.

\section{Results Study population}

The study population included a total of 864 premenopausal White women: 286 breast cancer patients diagnosed between 2006 and 2013 and 578 control 
participants selected between 2009 and 2014. The age of the patients ranged from 21 to 49 years (median age: 44.5 years, interquartile range: $41-49$ ), and that of the control participants ranged from 23 to 49 years (median age: 44 years, interquartile range: $41-49$ ).

Pairing showed no differences with respect to each 5-year age group $(P=0.90)$.

\section{Reproductive factors and cancer risk in premenopausal women}

As shown in Table 1, reproductive factors did not significantly relate to the risk of breast cancer in univariate analysis. The mean age at menarche was $12.33 \pm 1.49$ years in the case group and $12.51 \pm 1.58$ years in the control group, with no differences on statistical analysis $(P=0.11)$; the number of births was similar in the two groups: $69.2 \%$ of parous women in the case group and $67.5 \%$ of parous women in the control group $(P=0.60)$. The mean age at first full-term pregnancy was $28.88 \pm 5.51$ years among the patients and $28.78 \pm 5.25$ years among the control participants $(P=0.69)$. Therefore, the above-mentioned reproductive factors were not associated with the risk of breast cancer in premenopausal women.

Breastfeeding and cancer risk in premenopausal women The distribution of risk factors among premenopausal women with breast cancers compared with the controls is shown in Table 1 . In the multivariate analysis, parous women who breastfed for up to 12 months were at a lower risk of developing breast cancer (estimated OR: 0.41, 95\% CI: $0.24-0.70, \quad P=0.0009$ ). Women who had breastfed for more than 12 months showed an increase in the risk of breast cancer (OR: 2.32, 95\% CI: 1.26-4.34, $P=0.007)$. The other reproductive factors were not significantly related to the risk of breast cancers (Table 2).

\section{Reproductive factors and molecular subtypes of breast cancer}

To determine whether reproductive factors were associated with different molecular subtypes of breast cancer, 280 out of 286 breast cancer archive tissue samples were analyzed: $21(8 \%)$ in-situ carcinoma, $117(42 \%)$ luminal A tumors, 94 (33\%) luminal B tumors, 15 (5\%) HER2 + tumors, and $33(12 \%)$ triple-negative cancers. We excluded six cases: two because of missing data and four because they did not fulfill the St. Gallen classification criteria. For Ki-67 status, we chose the threshold greater than or equal to $20 \%$, which is used widely by pathologists (Goldhirsch et al., 2013). We compared this classification of our cohort with that suggested by Maisonneuve et al. (2014): only one of our cases of luminal A was classifiable as luminal B and one of our cases of luminal B was classifiable as luminal A. Thus, our classification of the cohort did not differ significantly from that suggested by Maisonneuve et al. (2014). Moreover, in the analysis, patients with in-situ carcinoma
Table 1 Distribution of risk factors among women with breast cancer (cases) and unaffected women (controls) ${ }^{\mathrm{a}}$

\begin{tabular}{|c|c|c|c|}
\hline Characteristics & $\begin{array}{l}\text { Women with breast } \\
\text { cancer ( } 286 \text { cases) }\end{array}$ & $\begin{array}{l}\text { Unaffected women } \\
\text { (578 controls) }\end{array}$ & $P_{\text {trend }}$ \\
\hline \multicolumn{4}{|l|}{ Age } \\
\hline Mean \pm SD & $43.3 \pm 4.8$ & $43.5 \pm 4.23$ & 0.90 \\
\hline $\begin{array}{l}\text { Median (interquartile } \\
\text { range) }\end{array}$ & $44.5(41-49)$ & $44.0(41-49)$ & \\
\hline Range & $21-49$ & $23-49$ & \\
\hline Parity $[n(\%)]$ & & & 0.60 \\
\hline Nulliparous & $88(30.8)$ & $188(32.5)$ & \\
\hline Parous & $198(69.2)$ & $390(67.5)$ & \\
\hline \multicolumn{4}{|l|}{ Age at menarche $[n(\%)]$} \\
\hline Mean \pm SD & $12.3 \pm 1.5$ & $12.5 \pm 1.6$ & 0.11 \\
\hline$<12$ & $80(28.5)$ & $134(23.4)$ & 0.33 \\
\hline 12 & $80(28.5)$ & $158(27.6)$ & \\
\hline 13 & $63(22.4)$ & $149(26.0)$ & \\
\hline$\geq 14$ & $58(20.6)$ & $132(23.0)$ & \\
\hline $\mathrm{BMI}\left(\mathrm{kg} / \mathrm{m}^{2}\right)[n(\%)]$ & & & 0.34 \\
\hline$<18.5$ & $14(5.0)$ & $36(6.3)$ & \\
\hline $18.5-24.9$ & $199(71.3)$ & $407(70.7)$ & \\
\hline $25-29.9$ & $38(13.6)$ & $92(16.0)$ & \\
\hline$\geq 30$ & $28(10.0)$ & $41(7.0)$ & \\
\hline $\begin{array}{l}\text { Oral contraceptive use } \\
{[n(\%)]}\end{array}$ & & & 0.60 \\
\hline Never & $169(61.2)$ & $324(58.0)$ & \\
\hline $\begin{array}{l}\text { Current (at least for } \\
5 \text { years) }\end{array}$ & $34(12.3)$ & $69(12.3)$ & \\
\hline $\begin{array}{l}\text { Former (at least for } \\
5 \text { years) }\end{array}$ & $73(26.5)$ & $166(29.7)$ & \\
\hline \multicolumn{4}{|l|}{ Parity $[n(\%)]$} \\
\hline $\begin{array}{l}\text { Number of births } \\
(\text { mean } \pm \text { SD })\end{array}$ & $1.7 \pm 0.7$ & $1.7 \pm 0.8$ & 0.60 \\
\hline 0 & $88(30.8)$ & $188(32.5)$ & 0.59 \\
\hline 1 & $88(30.8)$ & $174(30.1)$ & \\
\hline 2 & $87(30.4)$ & $183(31.7)$ & \\
\hline$\geq 3$ & $23(8.0)$ & $33(5.7)$ & \\
\hline $\begin{array}{l}\text { Benign breast disease } \\
{[n(\%)]}\end{array}$ & & & $0.02^{*}$ \\
\hline Yes & $17(5.9)$ & $16(2.8)$ & \\
\hline No & $269(94.1)$ & $562(97.2)$ & \\
\hline $\begin{array}{l}\text { Family history of breast } \\
\text { cancer }\end{array}$ & & & 0.14 \\
\hline None & $160(58.2)$ & $350(65.1)$ & \\
\hline $\begin{array}{l}\text { First-degree familial } \\
\quad(<50 \text { years })\end{array}$ & $24(8.7)$ & $39(7.3)$ & \\
\hline Second-degree familial & $91(33.1)$ & $148(27.6)$ & \\
\hline Only parous women & $\begin{array}{c}\text { Women with } \\
\text { breast cancer (198 } \\
\text { cases) }\end{array}$ & $\begin{array}{l}\text { Unaffected } \\
\text { women (390 } \\
\text { controls) }\end{array}$ & $P_{\text {trend }}$ \\
\hline \multicolumn{4}{|c|}{ Age at first pregnancy $[n(\%)]$} \\
\hline Mean \pm SD & $28.9 \pm 5.5$ & $28.7 \pm 5.3$ & 0.51 \\
\hline$<25$ & $46(23.2)$ & $92(23.8)$ & 0.85 \\
\hline $25-29$ & $58(29.3)$ & $120(31.1)$ & \\
\hline$\geq 30$ & $94(47.5)$ & $174(45.1)$ & \\
\hline Breastfeeding [n (\%)] & & & 0.13 \\
\hline Yes & $160(80.8)$ & $334(85.6)$ & \\
\hline No & $38(19.2)$ & $56(14.4)$ & \\
\hline \multicolumn{4}{|c|}{ Breastfeeding duration [n (\%)] } \\
\hline$<0.001^{\star}$ & $\leq 12$ months & $91(45.0)$ & \\
\hline & & & $\begin{array}{l}292 \\
(74.9)\end{array}$ \\
\hline$>12$ months & $69(34.8)$ & $42(10.7)$ & \\
\hline No & $38(19.2)$ & 56 (14.4) & \\
\hline
\end{tabular}

aNumbers do not add up to the total because of missing values. *Statistically significant.

and nonluminal breast cancer were not included because very few patients had these cancers.

We compared luminal A cases and luminal B cases separately with a group of controls selected among 578 
Table 2 Multivariable-adjusted odds ratios for breast cancer (only parous women)

\begin{tabular}{|c|c|c|c|}
\hline \multirow[b]{2}{*}{ Characteristics } & \multicolumn{2}{|c|}{$\begin{array}{l}\text { Women with breast cancer (198 } \\
\quad \text { cases) } \\
\text { Unaffected women ( } 390 \text { controls) }\end{array}$} & \multirow[b]{2}{*}{$P$-value } \\
\hline & $\mathrm{OR}^{\mathrm{a}}$ & $95 \% \mathrm{Cl}$ & \\
\hline \multicolumn{4}{|l|}{ Age at menarche } \\
\hline$<12$ & 1.01 & $0.60-1.69$ & 0.97 \\
\hline 12 & 1 & Reference & - \\
\hline 13 & 0.87 & $0.51-1.51$ & - \\
\hline$\geq 14$ & 0.81 & $0.45-1.43$ & - \\
\hline \multicolumn{4}{|l|}{$\mathrm{BMI}\left(\mathrm{kg} / \mathrm{m}^{2}\right)$} \\
\hline$<18.5$ & 0.5 & $0.17-1.29$ & 0.18 \\
\hline $18.5-24.9$ & 1 & Reference & - \\
\hline $25-29.9$ & 0.68 & $0.37-1.19$ & 0.18 \\
\hline$\geq 30$ & 1.25 & $0.58-2.62$ & 0.56 \\
\hline \multicolumn{4}{|l|}{ Oral contraceptive use } \\
\hline Never & 1.16 & $0.75-1.83$ & 0.51 \\
\hline Current (at least for 5 years) & 1 & Reference & - \\
\hline Former (at least for 5 years) & 1.15 & $0.59-2.22$ & 0.68 \\
\hline \multicolumn{4}{|l|}{ Benign breast disease } \\
\hline No & 1 & Reference & - \\
\hline Yes & 1.96 & $0.77-4.88$ & 0.22 \\
\hline \multicolumn{4}{|l|}{ Family history of breast cancer } \\
\hline None & 1 & Reference & - \\
\hline $\begin{array}{l}\text { First-degree familial } \\
\qquad(<50 \text { years })\end{array}$ & 1.37 & $0.64-2.83$ & 0.40 \\
\hline Second-degree familial & 1.31 & $0.84-2.02$ & 0.23 \\
\hline \multicolumn{4}{|l|}{ Breastfeeding duration } \\
\hline No & 1 & Reference & - \\
\hline$\leq 12$ months & 0.41 & $0.24-0.70$ & $<0.001^{*}$ \\
\hline$>12$ months & 2.32 & $1.26-4.34$ & $0.007^{*}$ \\
\hline
\end{tabular}

$\mathrm{Cl}$, confidence interval; OR, odds ratio.

${ }^{a}$ Odds ratios are adjusted for age, age at menarche, $\mathrm{BMI}$, oral contraceptive use, benign breast disease, and family history of breast cancer.

*Statistically significant.

negative women of the study, respectively, always matched by age. Tables 3 and 4 show the distribution of risk factors among luminal A and luminal B cases compared with the controls. In the multivariate analysis, reproductive factors were not correlated significantly with luminal A cancers (Table 5). In contrast, breastfeeding for up to 12 months did show a significant protective role for luminal B breast cancers (OR: 0.22, 95\% CI: 0.09-0.59, $P=0.002$ ), and, more interestingly, it was the only reproductive factor that was statistically significant in the multivariate analysis (Table 6). However, breastfeeding for more than 12 months was not a protective factor in both luminal A and luminal B molecular subtypes (NS).

\section{Discussion}

Our study analyzed the role of breastfeeding and reproductive factors in the risk of breast cancer in a homogenous selected population of White premenopausal women living in a country with a high risk of breast cancer, focusing on luminal molecular phenotypes, the most frequent cancers occurring in the population sample $(75 \%)$.

Parity was shown to be a protective factor for breast cancer in postmenopausal women, but not in premenopausal women (Clavel-Chapelon, 2002). Accordingly, our results confirm that in premenopausal women, the parity did not
Table 3 Distribution of risk factors among women with luminal A breast cancer and unaffected women ${ }^{a}$

\begin{tabular}{|c|c|c|c|}
\hline Characteristics & $\begin{array}{c}\text { Women with luminal A } \\
\text { breast cancer } \\
(N=117)\end{array}$ & $\begin{array}{l}\text { Unaffected women } \\
\text { (controls) }(N=234)\end{array}$ & $P_{\text {trend }}$ \\
\hline \multicolumn{4}{|l|}{ Age } \\
\hline Mean \pm SD & $44.4 \pm 4.4$ & $44.0 \pm 4.2$ & \\
\hline $\begin{array}{l}\text { Median (interquartile } \\
\text { range) }\end{array}$ & $45(43-48)$ & $45(42-47)$ & 0.37 \\
\hline Range & $25-49$ & $23-49$ & \\
\hline Parity $[n(\%)]$ & & & 0.29 \\
\hline Nulliparous & $32(27.4)$ & 77 (32.9) & \\
\hline Parous & $85(72.6)$ & $157(67.1)$ & \\
\hline \multicolumn{4}{|l|}{ Age at menarche $[n(\%)]$} \\
\hline Mean \pm SD & $12.4 \pm 1.5$ & $12.3 \pm 1.5$ & 0.73 \\
\hline$<12$ & $35(30.2)$ & $63(27.0)$ & 0.20 \\
\hline 12 & $27(23.4)$ & $64(27.5)$ & \\
\hline 13 & $27(23.4)$ & 59 (25.3) & \\
\hline$\geq 14$ & $27(23.4)$ & $47(20.2)$ & \\
\hline $\mathrm{BMI}\left(\mathrm{kg} / \mathrm{m}^{2}\right)[n(\%)]$ & & & 0.75 \\
\hline$<18.5$ & $7(6.1)$ & $13(5.6)$ & \\
\hline $18.5-24.9$ & $82(71.3)$ & $160(68.4)$ & \\
\hline $25-29.9$ & $15(13.0)$ & $41(17.5)$ & \\
\hline$\geq 30$ & $11(9.6)$ & $20(8.5)$ & \\
\hline $\begin{array}{l}\text { Oral contraceptive use } \\
{[n(\%)]}\end{array}$ & & & 0.19 \\
\hline Never & $64(57.1)$ & $137(60.9)$ & \\
\hline $\begin{array}{l}\text { Current (at least for } \\
5 \text { years) }\end{array}$ & $18(16.1)$ & $21(9.3)$ & \\
\hline $\begin{array}{l}\text { Former (at least for } \\
5 \text { years) }\end{array}$ & $30(26.8)$ & $67(29.8)$ & \\
\hline \multicolumn{4}{|l|}{ Parity $[n(\%)]$} \\
\hline $\begin{array}{l}\text { Number of births } \\
\text { (mean } \pm \text { SD) }\end{array}$ & $1.7 \pm 0.7$ & $1.6 \pm 0.6$ & 0.65 \\
\hline 0 & $32(27.4)$ & 77 (32.9) & 0.52 \\
\hline 1 & 39 (33.3) & 35 (32.5) & \\
\hline 2 & $36(30.8)$ & $31(29.5)$ & \\
\hline$\geq 3$ & $10(8.5)$ & $9(5.2)$ & \\
\hline $\begin{array}{l}\text { Benign breast disease } \\
{[n(\%)]}\end{array}$ & & & 0.19 \\
\hline Yes & $7(6.0)$ & $7(3.0)$ & \\
\hline No & $110(94.0)$ & $227(97.0)$ & \\
\hline $\begin{array}{l}\text { Family history of breast } \\
\text { cancer }[n(\%)]\end{array}$ & & & 0.22 \\
\hline $\begin{array}{l}\text { First-degree familial } \\
\quad(<50 \text { years })\end{array}$ & $7(6.3)$ & $19(9.5)$ & \\
\hline $\begin{array}{l}\text { Second-degree } \\
\text { familial }\end{array}$ & $43(38.4)$ & 59 (29.5) & \\
\hline None & $62(55.4)$ & $122(61.0)$ & \\
\hline Only parous women & $\begin{array}{l}\text { Women with } \\
\text { luminal } A \\
\text { breast } \\
\text { cancer } \\
(N=85)\end{array}$ & $\begin{array}{l}\text { Unaffected women } \\
\text { (controls) }(N=157)\end{array}$ & $P_{\text {trend }}$ \\
\hline \multicolumn{4}{|c|}{ Age at first pregnancy $[n(\%)]$} \\
\hline Mean \pm SD & $29.7 \pm 5.7$ & $28.7 \pm 5.2$ & 0.59 \\
\hline$<25$ & $16(18.8)$ & $36(23.4)$ & 0.30 \\
\hline $25-29$ & $22(25.9)$ & $49(31.8)$ & \\
\hline$\geq 30$ & 47 (55.3) & $69(44.8)$ & \\
\hline Breastfeeding [n (\%)] & & & 0.90 \\
\hline Yes & $72(84.7)$ & $132(84.0)$ & \\
\hline No & $13(15.3)$ & $25(16.0)$ & \\
\hline \multicolumn{4}{|c|}{$\begin{array}{l}\text { Breastfeeding duration }[n(\%)] \\
<0.001^{*}\end{array}$} \\
\hline$\leq 12$ months & $46(54.1)$ & $115(73.2)$ & \\
\hline$>12$ months & $26(30.6)$ & $17(10.8)$ & \\
\hline No & $13(15.3)$ & $25(16.0)$ & \\
\hline
\end{tabular}

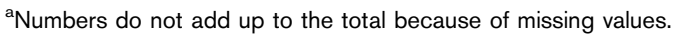
*Statistically significant.

reduce the risk of breast cancer. In contrast to the results reported by other authors, we cannot conclude that the lack of a protective effect of multiparity is because of the low 
Table 4 Distribution of risk factors among women with luminal B breast cancer and unaffected women ${ }^{\mathrm{a}}$

\begin{tabular}{|c|c|c|c|}
\hline Characteristics & $\begin{array}{c}\text { Women with luminal } \\
\text { B breast cancer } \\
(N=94)\end{array}$ & $\begin{array}{r}\text { Unaffected women } \\
\text { (controls) }(N=188)\end{array}$ & $P_{\text {trend }}$ \\
\hline \multicolumn{4}{|l|}{ Age } \\
\hline Mean \pm SD & $42.8 \pm 4.9$ & $42.8 \pm 5.4$ & \\
\hline $\begin{array}{l}\text { Median (interquartile } \\
\text { range) }\end{array}$ & $44(40-46)$ & $44(41-47)$ & 0.72 \\
\hline Range & $21-49$ & $21-49$ & \\
\hline Parity $[n(\%)]$ & & & 0.21 \\
\hline Nulliparous & $27(28.7)$ & $68(36.2)$ & \\
\hline Parous & $67(71.3)$ & $120(63.8)$ & \\
\hline \multicolumn{4}{|l|}{ Age at menarche $[n(\%)]$} \\
\hline Mean \pm SD & $12.4 \pm 1.5$ & $12.5 \pm 1.5$ & 0.28 \\
\hline$<12$ & $24(26.1)$ & $41(21.8)$ & 0.51 \\
\hline 12 & 29 (31.5) & $57(30.3)$ & \\
\hline 13 & $18(19.6)$ & $52(27.7)$ & \\
\hline$\geq 14$ & $21(22.8)$ & $38(20.2)$ & \\
\hline $\mathrm{BMI}\left(\mathrm{kg} / \mathrm{m}^{2}\right)$ & & & 0.58 \\
\hline$<18.5$ & $4(4.3)$ & $15(7.9)$ & \\
\hline $18.5-24.9$ & $66(71.7)$ & $134(71.3)$ & \\
\hline $25-29.9$ & $14(15.2)$ & $28(14.9)$ & \\
\hline$\geq 30$ & $8(8.8)$ & $11(5.9)$ & \\
\hline Oral contraceptive use & & & 0.06 \\
\hline Never & $60(65.9)$ & $93(51.1)$ & \\
\hline $\begin{array}{l}\text { Current (at least for } \\
5 \text { years) }\end{array}$ & $9(9.9)$ & $31(17.0)$ & \\
\hline $\begin{array}{l}\text { Former (at least for } \\
5 \text { years) }\end{array}$ & $22(24.2)$ & $58(31.9)$ & \\
\hline \multicolumn{4}{|l|}{ Parity } \\
\hline $\begin{array}{l}\text { Number of births } \\
\text { (mean } \pm \text { SD) }\end{array}$ & $1.7 \pm 0.7$ & $1.7 \pm 0.6$ & 0.80 \\
\hline 0 & $27(28.7)$ & $68(36.2)$ & 0.25 \\
\hline 1 & $31(33.0)$ & $44(23.4)$ & \\
\hline 2 & $28(29.8)$ & $64(34.0)$ & \\
\hline$\geq 3$ & $8(8.5)$ & $12(6.4)$ & \\
\hline $\begin{array}{l}\text { Benign breast disease } \\
{[n(\%)]}\end{array}$ & & & 0.38 \\
\hline Yes & $3(3.2)$ & $3(1.6)$ & \\
\hline No & $91(96.8)$ & $185(98.4)$ & \\
\hline $\begin{array}{l}\text { Family history of breast } \\
\text { cancer }[n(\%)]\end{array}$ & & & 0.26 \\
\hline $\begin{array}{l}\text { First-degree familial } \\
(<50 \text { years })\end{array}$ & $7(7.7)$ & $15(8.0)$ & \\
\hline $\begin{array}{l}\text { Second-degree } \\
\text { familial }\end{array}$ & $27(29.7)$ & $42(22.3)$ & \\
\hline None & $57(62.6)$ & $131(69.7)$ & \\
\hline Only parous women & $\begin{array}{c}\text { Women with luminal } \\
\text { B breast cancer } \\
(N=67)\end{array}$ & $\begin{array}{l}\text { Unaffected women } \\
\text { (controls) }(N=120)\end{array}$ & $P_{\text {trend }}$ \\
\hline \multicolumn{4}{|c|}{ Age at first pregnancy $[n(\%)]$} \\
\hline Mean \pm SD & $28.2 \pm 5.3$ & $28.4 \pm 4.9$ & 0.65 \\
\hline$<25$ & $16(22.9)$ & $33(27.5)$ & 0.75 \\
\hline $25-29$ & $24(35.8)$ & $37(30.8)$ & \\
\hline$\geq 30$ & $27(40.3)$ & $50(41.7)$ & \\
\hline Breastfeeding [ $n(\%)]$ & & & $0.01^{*}$ \\
\hline Yes & $51(76.1)$ & $108(90.0)$ & \\
\hline No & $16(23.9)$ & $12(10.0)$ & \\
\hline $\begin{array}{l}\text { Breastfeeding } \\
\text { duration [n (\%)] }\end{array}$ & & & $<0.001^{*}$ \\
\hline$\leq 12$ months & 25 (37.3) & $97(80.8)$ & \\
\hline$>12$ months & $26(38.8)$ & $11(9.2)$ & \\
\hline No & $16(22.9)$ & $12(10.0)$ & \\
\hline
\end{tabular}

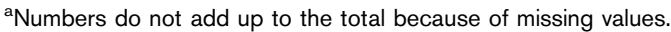
*Statistically significant.

number of multiparous young women: the births were not correlated to the risk of breast cancer in a statistically significant sample of multiparous women. Moreover, we found that younger age at first full-term pregnancy did not confer protection; no significant increase in the risk of
Table 5 Multivariable-adjusted odds ratios for breast cancer (only parous women)

\begin{tabular}{|c|c|c|c|}
\hline \multirow[b]{2}{*}{ Characteristics } & \multicolumn{2}{|c|}{$\begin{array}{l}\text { Women with luminal A breast cancer } \\
\qquad(N=85) \\
\text { Unaffected women (controls) } \\
\qquad(N=157)\end{array}$} & \multirow[b]{2}{*}{$P$-value } \\
\hline & $\mathrm{OR}^{\mathrm{a}}$ & $95 \% \mathrm{Cl}$ & \\
\hline \multicolumn{4}{|l|}{ Age at menarche } \\
\hline$<12$ & 1.27 & $0.57-2.85$ & 0.56 \\
\hline 12 & 1 & Reference & - \\
\hline 13 & 0.95 & $0.39-2.3$ & 0.90 \\
\hline$\geq 14$ & 1.1 & $0.48-2.73$ & 0.84 \\
\hline \multicolumn{4}{|l|}{$\mathrm{BMI}\left(\mathrm{kg} / \mathrm{m}^{2}\right)$} \\
\hline$<18.5$ & 0.73 & $0.14-3.0$ & 0.67 \\
\hline $18.5-24.9$ & 1 & Reference & - \\
\hline $25-29.9$ & 0.46 & $0.16-1.15$ & 0.11 \\
\hline$\geq 30$ & 1.05 & $0.32-3.15$ & 0.93 \\
\hline \multicolumn{4}{|l|}{ Oral contraceptive use } \\
\hline Never & 1.13 & $0.57-2.25$ & 0.73 \\
\hline Current (at least for 5 years) & 1 & Reference & - \\
\hline Former (at least for 5 years) & 2.09 & $0.74-6.0$ & 0.16 \\
\hline \multicolumn{4}{|l|}{ Benign breast disease } \\
\hline No & 1 & Reference & - \\
\hline Yes & 3.64 & $0.92-5.0$ & 0.06 \\
\hline \multicolumn{4}{|l|}{ Family history of breast cancer } \\
\hline None & 1 & Reference & - \\
\hline $\begin{array}{l}\text { First-degree familial } \\
\qquad(<50 \text { years })\end{array}$ & 0.82 & $0.23-2.60$ & 0.74 \\
\hline Second-degree familial & 1.2 & $0.61-2.35$ & 0.58 \\
\hline \multicolumn{4}{|l|}{ Breastfeeding duration } \\
\hline No & 1 & Reference & - \\
\hline$\leq 12$ months & 0.55 & $0.18-1.65$ & 0.28 \\
\hline$>12$ months & 2.43 & $0.87-7.01$ & 0.09 \\
\hline
\end{tabular}

$\mathrm{Cl}$, confidence interval; OR, odds ratio.

${ }^{a}$ Odds ratios are adjusted for age, age at menarche, BMl, oral contraceptive use, benign breast disease, and family history of breast cancer.

cancer was found both for multiparity and for age at menarche. Interestingly, familial history of breast cancer of both first and second grade in our cohort of premenopausal women was not significantly related to the risk of breast cancer. This can be attributed to the lack of a significant number of cases with first-grade familial history of breast or ovarian cancer at a young age, which is highly related to an increased risk of breast cancer (six cases).

Literature data show that reproductive factors are related to the molecular subtypes of breast cancer (Althuis $e t$ al., 2004; Ursin et al., 2005; Tamimi et al., 2012); hormonal factors have a greater impact on luminal than nonluminal types of breast cancer, the risk being predominantly influenced by hormonal mechanisms (Anderson, 2002; Dickson and Stancel, 2000; Anderson et al., 2014). Hormonal risk factors such as parity and early age at first birth seem to relate positively to luminal subtypes compared with nonluminal tumors (Ma et al., 2006). Previous studies have suggested a protective effect of multiparity and young age at first full-term pregnancy on breast cancer, but this protective effect appears to be different among the various breast cancer receptor statuses: multiparity seems to exert a protective effect on $\mathrm{ER}+/ \mathrm{PR}+$ tumors, but not on receptor-negative ones (Yoo et al., 1997; Cotterchio et al., 2003; Ursin et al., 2005; Kwan et al., 2009). Recently, a large population-based prospective 
Table 6 Multivariable-adjusted odds ratios for breast cancer (only parous women)

\begin{tabular}{|c|c|c|c|}
\hline \multirow[b]{2}{*}{ Characteristics } & \multicolumn{2}{|c|}{$\begin{array}{l}\text { Women with luminal B breast cancer } \\
\qquad(N=67) \\
\text { Unaffected women (controls) } \\
(N=120)\end{array}$} & \multirow[b]{2}{*}{$P$-value } \\
\hline & $\mathrm{OR}^{\mathrm{a}}$ & $95 \% \mathrm{Cl}$ & \\
\hline \multicolumn{4}{|l|}{ Age at menarche } \\
\hline$<12$ & 0.99 & $0.38-2.60$ & 0.99 \\
\hline 12 & 1 & Reference & - \\
\hline 13 & 0.81 & $0.28-2.29$ & 0.70 \\
\hline$\geq 14$ & 1.17 & $0.42-3.24$ & 0.76 \\
\hline \multicolumn{4}{|l|}{ BMI $\left(\mathrm{kg} / \mathrm{m}^{2}\right)$} \\
\hline$<18.5$ & 0.33 & $0.04-1.84$ & 0.25 \\
\hline $18.5-24.9$ & 1 & Reference & - \\
\hline $25-29.9$ & 0.91 & $0.32-2.39$ & 0.84 \\
\hline$\geq 30$ & 1.20 & $0.32-4.49$ & 0.78 \\
\hline \multicolumn{4}{|l|}{ Oral contraceptive use } \\
\hline Never & 1.42 & $0.61-3.28$ & 0.42 \\
\hline Current (at least for 5 years) & 1 & Reference & - \\
\hline Former (at least for 5 years) & 0.62 & $0.17-2.05$ & 0.44 \\
\hline \multicolumn{4}{|l|}{ Benign breast disease } \\
\hline No & 1 & Reference & - \\
\hline Yes & 1.52 & $0.15-11.60$ & 0.69 \\
\hline \multicolumn{4}{|l|}{ Family history of breast cancer } \\
\hline None & 1 & Reference & - \\
\hline $\begin{array}{l}\text { First-degree familial } \\
\qquad(<50 \text { years })\end{array}$ & 1.28 & $0.33-4.59$ & 0.70 \\
\hline Second-degree familial & 1.29 & $0.55-2.96$ & 0.54 \\
\hline \multicolumn{4}{|l|}{ Breastfeeding duration } \\
\hline No & 1 & Reference & - \\
\hline$\leq 12$ months & 0.22 & $0.09-0.59$ & $0.002^{*}$ \\
\hline$>12$ months & 2.15 & $0.70-6.78$ & 0.18 \\
\hline
\end{tabular}

$\mathrm{Cl}$, confidence interval; OR, odds ratio.

${ }^{a}$ Odds ratios are adjusted for age, age at menarche, $\mathrm{BMI}$, oral contraceptive use, benign breast disease, and family history of breast cancer.

study with long-term follow-up (Horn et al., 2014) confirmed that the associations of reproductive factors with the risk of breast cancer may vary according to different molecular subtypes. They found that early menarche, older age at first birth, and low parity were associated with an increased risk for luminal breast cancer, whereas for the nonluminal type, there were no associations.

We found that age at menarche, parity, and age at first full-term pregnancy did not affect luminal A and luminal B breast cancers in our cohort of premenopausal women.

Reports of large series observed a protective effect in the cohorts of both premenopausal and postmenopausal women (Ursin et al., 2005; Kobayashi et al., 2012; Anderson et al., 2014). In the population of premenopausal women, breastfeeding was found to be protective in some studies (Collaborative Group on Hormonal Factors in Breast Cancer, 2002; Shema et al., 2007; Nelson et al., 2012; Warner et al., 2013; Trentham-Dietz et al., 2014), but not in others (Tessaro et al., 2003; Shantakumar et al., 2007; Butt et al., 2014). Several studies (Phipps et al., 2008; Butt et al., 2014; Horn et al., 2014) and meta-analyses (Collaborative Group on Hormonal Factors in Breast Cancer, 2002; Althuis et al., 2004) were published to better clarify the effect of breastfeeding and reproductive factors on the incidence of different breast cancer subtypes, and the majority of published data were from postmenopausal women (Phipps et al., 2008; Horn et al., 2014) or a large study population of both premenopausal and postmenopausal breast cancer patients (Kelsey et al., 1993; Ursin et al., 2005).

On multivariate analysis, we found that breastfeeding up to 12 months reduced the risk of luminal $\mathrm{B}$ but not luminal A breast cancer in this selected population. Breastfeeding was found to protect against both $\mathrm{ER}+1$ $\mathrm{PR}+$ and ER - /PR - tumors (Huang et al., 2000a; Ursin et al., 2005; Ma et al., 2006; Kwan et al., 2009). Other studies showed a reduced risk of basal-like triple negative breast cancer among women who breastfed for more than 4 months in African-American and White women, or in African-American women only (Millikan et al., 2008; Palmer et al., 2014). It is noteworthy that, in our study, among luminal cancers, only luminal B cancer was significantly related to breastfeeding. It might be conceivable, as suggested by several studies (Ma et al., 2006; Phipps et al., 2008; Kobayashi et al., 2012), that breastfeeding acts through different hormonal mechanisms than parity, thus determining the risk for different subtypes of breast cancer. In this respect, it cannot be excluded that the same factors might also play a role in determining the tumor aggressiveness in those established cancers. However, we cannot support any conclusion on the incidence of HER2 + or triple-negative cancers and breastfeeding because of the low number of cases.

Unexpectedly, overall, breastfeeding for more than 12 months was not linked to breast cancer risk reduction in our population of premenopausal women compared with those who never breastfed, for both luminal A and B cancers (Tables 5 and 6). These findings seem to be in contrast to the results of a large meta-analysis (Collaborative Group on Hormonal Factors in Breast Cancer, 2002) and other reports (Shema et al., 2007; Nelson et al., 2012; Trentham-Dietz et al., 2014) that indicate that the longer women breastfeed, the more they are protected against breast cancer. However, we would like to report observations of two other studies (Huang et al., 2000b; Yang and Jacobsen, 2008) that did not show an association between increased duration of lactation and reduction of the risk of breast cancer. Moreover, Gustbee et al. (2013) reported a high risk for early events of breast cancer for a total duration of breastfeeding of more than 12 months, perhaps because of the proproliferation effect of prolactin. Furthermore, three case-control studies showed that longer breastfeeding duration was a risk factor for breast cancers: a multicenter case-control study carried out in Italy (Negri et al., 1996), a Turkish study (Ulusoy et al., 2010), and a recent Serbian study (Ilic et al., 2015). A large Italian study (Negri et al., 1996) included premenopausal and postmenopausal women and excluded any appreciable protective role for 
lactation independent of the duration of breastfeeding. Ulusoy et al. (2010) investigated a population of both premenopausal and postmenopausal Turkish women, showing that a 10-month increase in breastfeeding increased the risk of breast cancer by $8 \%$ (OR: $1.008,95 \%$ CI: $1.003-1.012, P=0.0001)$. Nevertheless, this study does not indicate whether the OR has been adjusted for the other factors analyzed in a multivariate manner. Finally, Ilic et al. (2015) reported that in women living in Serbia, the risk of breast cancer increases with the duration of breastfeeding (OR: 3.44, 95\% CI: 1.15-10.24, $P=0.023$; for women who breastfed $>13$ months). Also, in this case, a sample of both premenopausal and postmenopausal women was analyzed. Our analysis indicates that breastfeeding for more than 12 months increases the risk of breast cancer, considering all breast cancer cases, but we cannot conclude on a general negative relation because of the fact that significance was not achieved on stratifying luminal A and luminal B cases.

In contrast to the results reported by these authors (Negri et al., 1996; Ulusoy et al., 2010; Ilic et al., 2015), we found lactation up to 12 months to be a protective factor for luminal B cancer and our study showed some differences: (a) our cohort was homogeneous and included premenopausal White women; (b) our results were obtained by matching one case with two controls $(1: 2$ case-control study); and (c) our study considered the molecular phenotype of breast cancers. It is noteworthy that we did not observe a reduction in the risk of breast cancer in relation to breastfeeding, but a less aggressive cancer in breastfeeding women that depends on the duration of lactation. In women with more than 12 months of lactation, the lack of breastfeeding protection was not expected. As in the majority of the literature, we considered the total duration of breastfeeding derived from the sum of the breastfeeding months for all children (if more than one) for each woman. To clarify this point, we analyzed whether there is a difference related to more than 12 months of lactation in women with one child ( 88 cases/ 174 controls). The results confirmed that breastfeeding for more than 12 months was not protective in multivariate analysis. The same results were found for the case group of women with two children. In this respect, we underline that, in our cohort of cases, the majority of women had one or two children (175 cases/357 controls). Thus, the results obtained on the total duration of breastfeeding appear to be consistent. We cannot exclude that breastfeeding for more than 12 months might be a protective factor or might contribute toward increasing the risk of breast cancer depending on the type of population analyzed (i.e. race). Although overall observations showed that breastfeeding for more than 12 months was not protective, further investigations on a higher number of cases are necessary.

In conclusion, this study of a homogeneous cohort of White premenopausal women showed no statistical associations between age at menarche, parity, age at first birth, and number of births and the risk of breast cancer. In contrast, the study supports evidence that, among luminal breast cancers, breastfeeding for up to 12 months is strongly protective against the more aggressive luminal $\mathrm{B}$ rather than the less aggressive luminal A type.

\section{Acknowledgements}

The authors thank Cristina Bottin and Anamaria Gheorghe-Guta for their important work in entering the questionnaire results in the database.

This work was supported by a grant of ' 5 per mille 2012' from Lega Italiana per la Lotta contro i Tumori (LILT), Italian Minister of Health.

\section{Conflicts of interest}

There are no conflicts of interest.

\section{References}

Althuis MD, Fergenbaum JH, Garcia-Closas M, Brinton LA, Madigan MP, Sherman ME (2004). Etiology of hormone receptor-defined breast cancer: a systematic review of the literature. Cancer Epidemiol Biomarkers Prev 13:1558-1568.

Anderson $E$ (2002). The role of oestrogen and progesterone receptors in human mammary development and tumorigenesis. Breast Cancer Res 4:197-201.

Anderson KN, Schwab RB, Martinez ME (2014). Reproductive risk factors and breast cancer subtypes: a review of the literature. Breast Cancer Res Treat 144:1-10.

Azim HA Jr, Partridge AH (2014). Biology of breast cancer in young women. Breast Cancer Res 16:427.

Butt S, Borgquist S, Anagnostaki L, Landberg G, Manjer J (2014). Breastfeeding in relation to risk of different breast cancer characteristics. BMC Res Notes 7:216.

Clavel-Chapelon F (2002). Differential effects of reproductive factors on the risk of pre- and postmenopausal breast cancer. Results from a large cohort of French women. Br J Cancer 86:723-727.

Collaborative Group on Hormonal Factors in Breast Cancer (2002). Breast cancer and breastfeeding: collaborative reanalysis of individual data from 47 epidemiological studies in 30 countries, including 50302 women with breast cancer and 96973 women without the disease. Lancet 360:187-195.

Cotterchio M, Kreiger N, Theis B, Sloan M, Bahl S (2003). Hormonal factors and the risk of breast cancer according to estrogen- and progesterone-receptor subgroup. Cancer Epidemiol Biomarkers Prev 12:1053-1060.

Dickson RB, Stancel GM (2000). Estrogen receptor-mediated processes in normal and cancer cells. J Natl Cancer Inst Monogr 27:135-145.

Ferlay J, Steliarova-Foucher E, Lortet-Tieulent J, Rosso S, Coebergh JW, Comber $\mathrm{H}$, et al. (2013). Cancer incidence and mortality patterns in Europe: estimates for 40 countries in 2012. Eur J Cancer 49:1374-1403.

Goldhirsch A, Winer EP, Coates AS, Gelber RD, Piccart-Gebhart M, Thürlimann B, Senn HJ, Panel members (2013). Personalizing the treatment of women with early breast cancer: highlights of the St Gallen International Expert Consensus on the Primary Therapy of Early Breast Cancer 2013. Ann Oncol 24:2206-2223.

Gustbée E, Anesten C, Markkula A, Simonsson M, Rose C, Ingvar C, Jernström H (2013). Excessive milk production during breast-feeding prior to breast cancer diagnosis is associated with increased risk for early events. Springerplus 2:298.

Horn J, Opdahl S, Engstrøm MJ, Romundstad PR, Tretli S, Haugen OA, et al. (2014). Reproductive history and the risk of molecular breast cancer subtypes in a prospective study of Norwegian women. Cancer Causes Control 25:881-889.

Huang WY, Newman B, Millikan RC, Conway K, Hulka BS, Schell MJ, Liu ET (2000a). Risk of breast cancer according to the status of HER-2/neu oncogene amplification. Cancer Epidemiol Biomarkers Prev 9:65-71.

Huang WY, Newman B, Millikan RC, Schell MJ, Hulka BS, Moorman PG (2000b). Hormone-related factors and risk of breast cancer in relation to estrogen receptor and progesterone receptor status. Am J Epidemiol 151:703-714.

Ilic M, Vlajinac H, Marinkovic J (2015). Breastfeeding and risk of breast cancer: case-control study. Women Health 55:778-794. 
Kelsey JL, Gammon MD, John EM (1993). Reproductive factors and breast cancer. Epidemiol Rev 15:36-47.

Kobayashi S, Sugiura H, Ando Y, Shiraki N, Yanagi T, Yamashita H, Toyama T (2012). Reproductive history and breast cancer risk. Breast Cancer 19:302-308.

Kwan ML, Kushi LH, Weltzien E, Maring B, Kutner SE, Fulton RS, et al. (2009). Epidemiology of breast cancer subtypes in two prospective cohort studies of breast cancer survivors. Breast Cancer Res 11:R31.

Ma H, Bernstein L, Pike MC, Ursin G (2006). Reproductive factors and breast cancer risk according to joint estrogen and progesterone receptor status: a meta-analysis of epidemiological studies. Breast Cancer Res 8:R43.

Maisonneuve P, Disalvatore D, Rotmensz N, Curigliano G, Colleoni M, Dellapasqua $S$, et al. (2014). Proposed new clinicopathological surrogate definitions of luminal $A$ and luminal $B$ (HER2-negative) intrinsic breast cancer subtypes. Breast Cancer Res 16:R65.

Millikan RC, Newman B, Tse CK, Moorman PG, Conway K, Dressler LG, et al. (2008). Epidemiology of basal-like breast cancer. Breast Cancer Res Treat 109:123-139.

Negri E, Braga C, La VC, Levi F, Talamini R, Franceschi S (1996). Lactation and the risk of breast cancer in an Italian population. Int J Cancer 67:161-164.

Nelson HD, Zakher B, Cantor A, Fu R, Griffin J, O'Meara ES, et al. (2012). Risk factors for breast cancer for women aged 40 to 49 years: a systematic review and meta-analysis. Ann Intern Med 156:635-648.

Palmer JR, Viscidi E, Troester MA, Hong CC, Schedin P, Bethea TN, et al. (2014). Parity, lactation, and breast cancer subtypes in African American women: results from the AMBER Consortium. J Nat/ Cancer Inst 106:pii: dju237.

Phipps Al, Malone KE, Porter PL, Daling JR, Li Cl (2008). Reproductive and hormonal risk factors for postmenopausal luminal, HER-2-overexpressing, and triple-negative breast cancer. Cancer 113:1521-1526.

Rotunno M, Sun X, Figueroa J, Sherman ME, Garcia-Closas M, Meltzer P, et al. (2014). Parity-related molecular signatures and breast cancer subtypes by estrogen receptor status. Breast Cancer Res 16:R74.

Shantakumar S, Terry MB, Teitelbaum SL, Britton JA, Millikan RC, Moorman PG, et al. (2007). Reproductive factors and breast cancer risk among older women. Breast Cancer Res Treat 102:365-374.

Shema L, Ore L, Ben-Shachar M, Haj M, Linn S (2007). The association between breastfeeding and breast cancer occurrence among Israeli Jewish women: a case control study. J Cancer Res Clin Oncol 133:539-546.
Siegel RL, Miller KD, Jemal A (2015). Cancer statistics, 2015. CA Cancer J Clin 65:5-29.

Tamimi RM, Colditz GA, Hazra A, Baer HJ, Hankinson SE, Rosner B, et al. (2012). Traditional breast cancer risk factors in relation to molecular subtypes of breast cancer. Breast Cancer Res Treat 131:159-167.

Tessaro S, Béria JU, Tomasi E, Victora CG (2003). Breastfeeding and breast cancer: a case-control study in Southern Brazil. Cad Saude Publica 19:1593-1601.

Trentham-Dietz A, Sprague BL, Hampton JM, Miglioretti DL, Nelson HD, Titus LJ, et al. (2014). Modification of breast cancer risk according to age and menopausal status: a combined analysis of five population-based case-control studies. Breast Cancer Res Treat 145:165-175.

Ulusoy C, Kepenekci I, Kose K, Aydintug S, Cam R (2010). Applicability of the Gail model for breast cancer risk assessment in Turkish female population and evaluation of breastfeeding as a risk factor. Breast Cancer Res Treat 120:419-424

Ursin G, Bernstein L, Lord SJ, Karim R, Deapen D, Press MF, et al. (2005). Reproductive factors and subtypes of breast cancer defined by hormone receptor and histology. $\mathrm{Br} J$ Cancer 93:364-371.

Warner ET, Colditz GA, Palmer JR, Partridge AH, Rosner BA, Tamimi RM (2013). Reproductive factors and risk of premenopausal breast cancer by age at diagnosis: are there differences before and after age 40? Breast Cancer Res Treat 142:165-175.

Wolff AC, Hammond ME, Schwartz JN, Hagerty KL, Allred DC, Cote RJ, et al. (2007). American Society of Clinical Oncology/College of American Pathologists Guideline Recommendations for human epidermal growth factor receptor 2 testing in breast cancer. J Clin Oncol 25:118-145.

Yang L, Jacobsen KH (2008). A systematic review of the association between breastfeeding and breast cancer. J Womens Health (Larchmt) 17:1635-1645.

Yoo KY, Tajima K, Miura S, Takeuchi T, Hirose K, Risch H, et al. (1997). Breast cancer risk factors according to combined estrogen and progesterone receptor status: a case-control analysis. $A m, J$ Epidemiol 146: 307-314.

Zucchetto A, Serraino D, Dal ML, Birri S, Francisci S, Zigon G, De AR (2013). Cancer estimates up to 2015 in Friuli Venezia Giulia. Tumori 99:318-326. 